CORRECTION

https://doi.org/10.1038/s41586-019-1627-z

\title{
Publisher Correction: An actin-based viscoplastic lock ensures progressive body-axis elongation
}

Alicia Lardennois, Gabriella Pásti, Teresa Ferraro, Flora Llense, Pierre Mahou, Julien Pontabry, David Rodriguez,

Samantha Kim, Shoichiro Ono, Emmanuel Beaurepaire, Christelle Gally \& Michel Labouesse

Correction to: Nature https://doi.org/10.1038/s41586-019-1509-4, published online 28 August 2019.

In Figs. $1 \mathrm{c}$ and $3 \mathrm{a}$ of this Letter, the genotype should have read 'Pdpy-7::LifeAct::GFP' instead of 'Ppdy-7::LifeAct::GFP'. In addition, the black box in Extended Data Fig. 1d should have shown the text 'spc-1(RNAi) pix-1(gk416)'. These errors have been corrected online. 